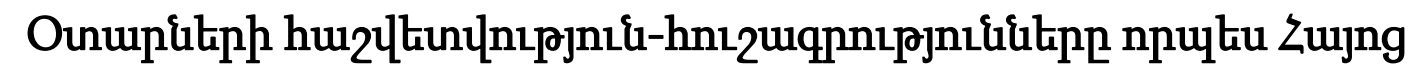

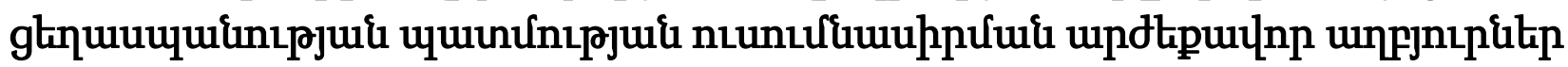

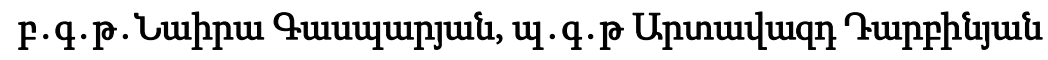 \\ Eplumip upkunulyui hưưuuupui \\ El. hnuun: gasparyannaira59@gmail.com
}

Paper received 22.07.19; Accepted for publication 09.08.19.

\begin{abstract}
https://doi.org/10.31174/SEND-HS2019-205VII34-11
\end{abstract}

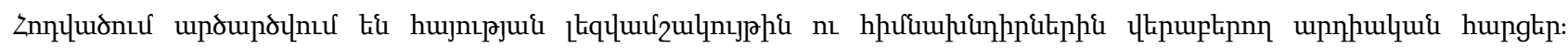

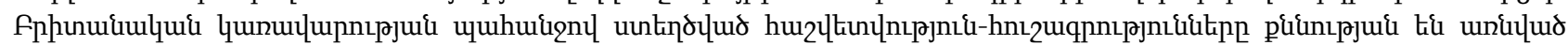

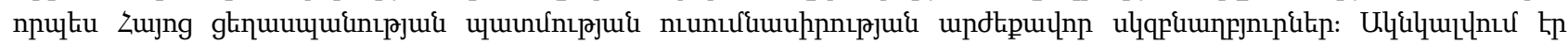

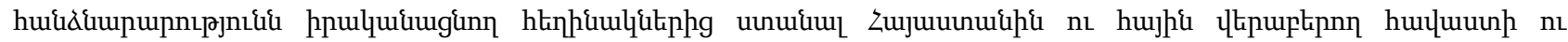

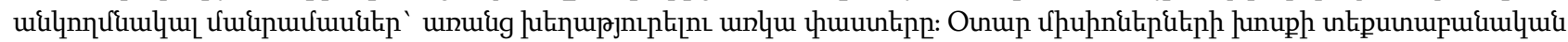

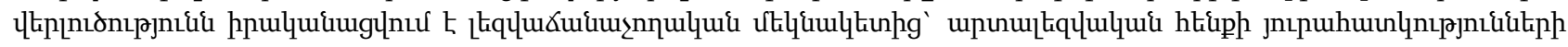

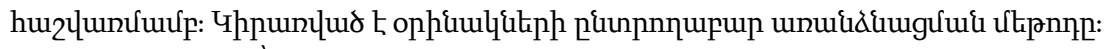

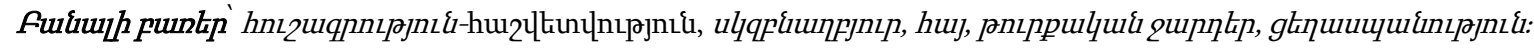

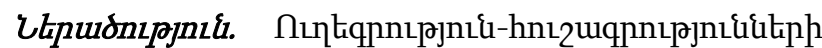

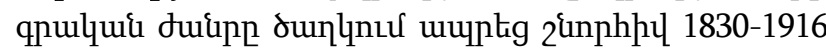

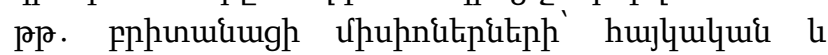

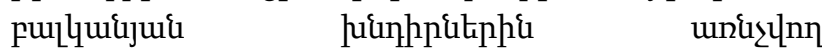

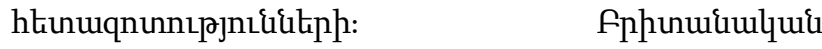

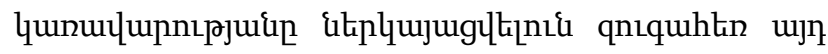

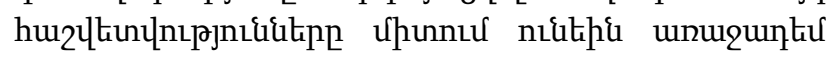
umpnlnnpjuian ppuqtilis pnenpulquis

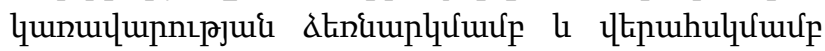

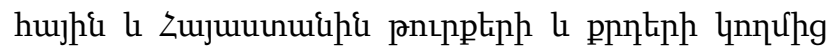

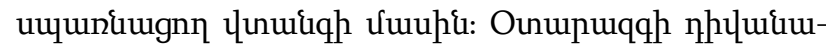

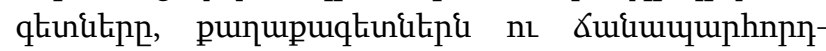

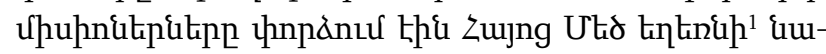

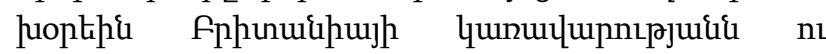

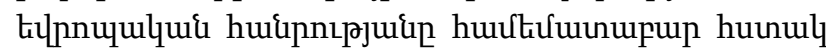

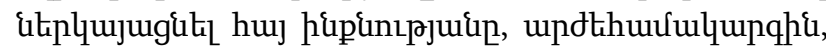

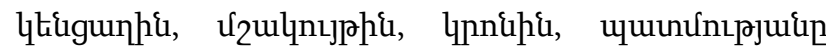

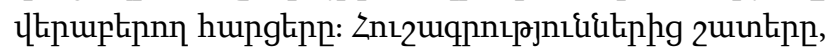

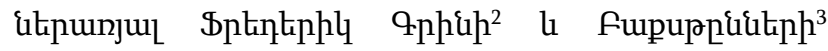

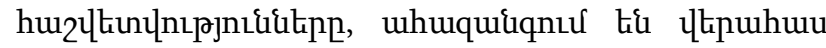

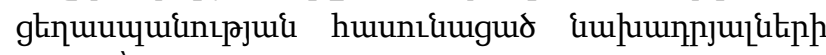

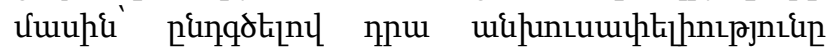

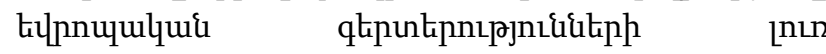

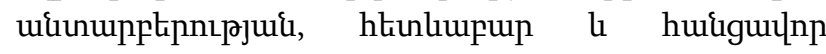

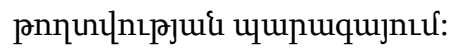

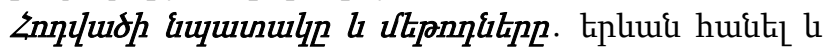

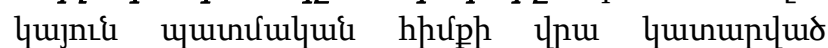

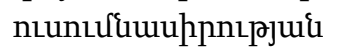

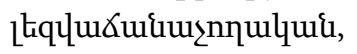
27quiuulqutipnư

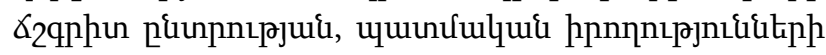

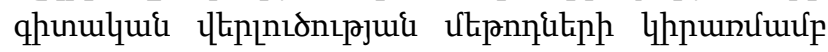

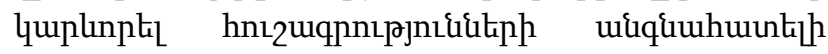

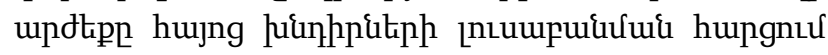

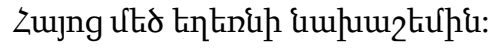

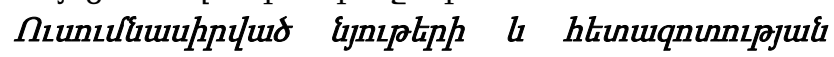

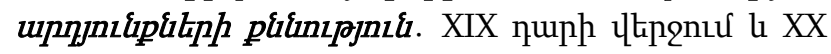
nuph ulqquitiphi hujng nnptenqnцрјui

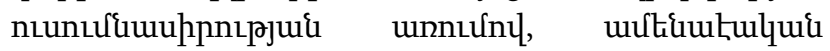

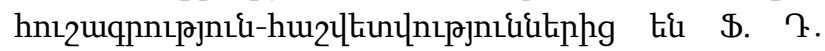
9phiu «Armenian Massacres and Turkish Tyrany»UU乙-

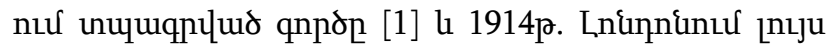

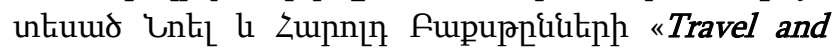
Politics in Armenia» qhppe [2]: Uútphlyuhuquunul

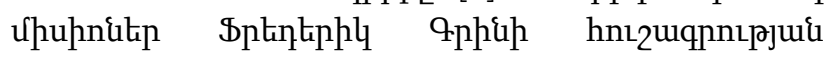

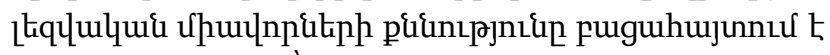

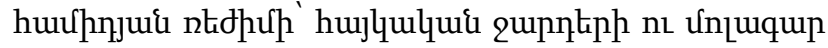

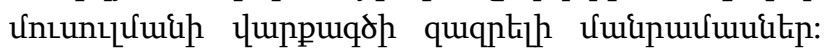

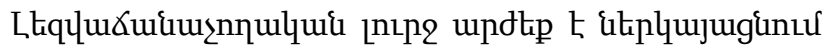

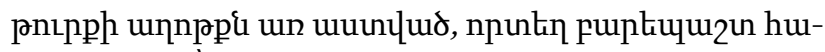

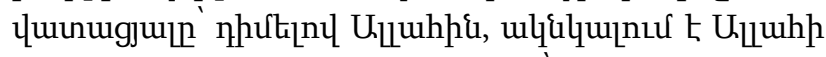

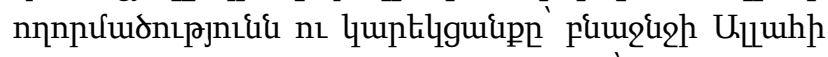

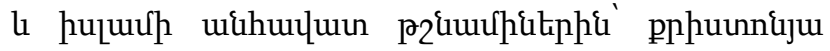

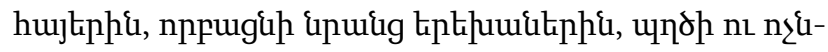

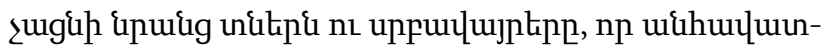

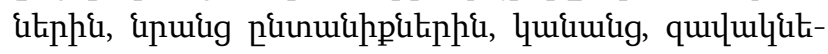

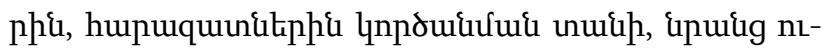

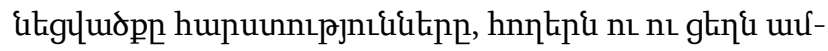

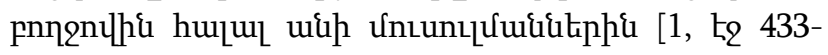
434]:

Unnpph punuph uquhwiquunhpulqui dlap

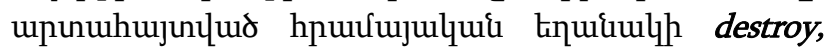
defile, make, cause pujuålitiph lhpunưứ,

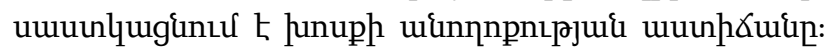

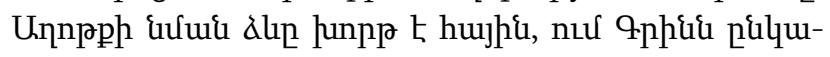

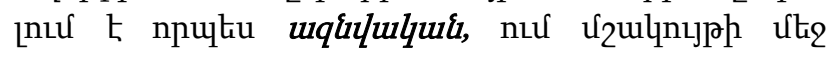
purgulqujnud t puppunnu tquidulynl hungtpp

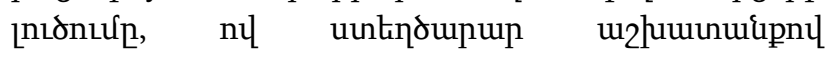




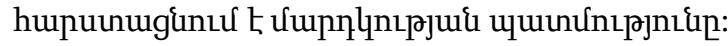

"I seek refuge with Allah from Satan, the accursed. In the name of Allah the Compassionate, the Merciful! O Lord of all Creatures! $O$ Allah! Destroy the infidels and polytheists, thine enemies, the enemies of the religion! $O$ Allah! Make their children orphans, and defile their abodes! Cause their feet to slip; them and their families, their households and their women, their children and their relations by marriage, their brothers and their friends, their possessions and their race, their wealth and their lands, as booty to the Moslems, $O$ Lord of all Creatures!" [1, to 434]

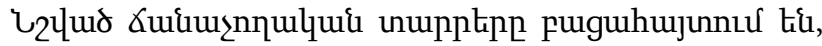

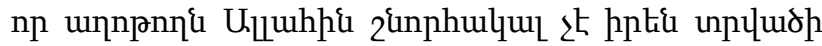

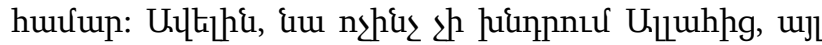

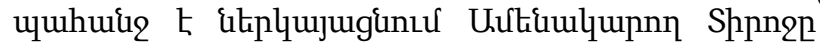

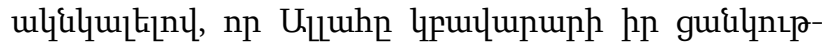

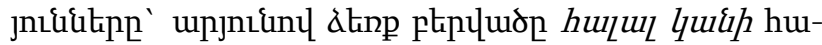

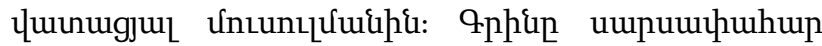

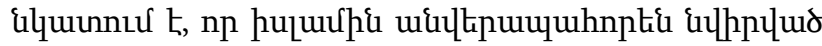

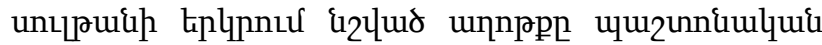

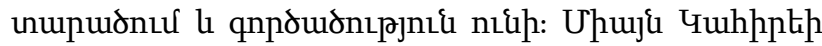
Azhar hưưuumpuintu tplph unuppten fuukphg hu-

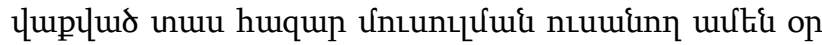

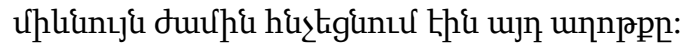

Unuehis hujugphg uifultn pluugn prayer - unnpp l student - nuuwinn qnjulquikilinn, hujuniultinnl pugu-

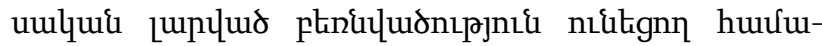

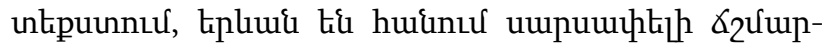

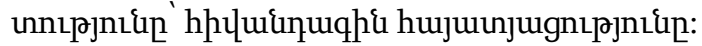

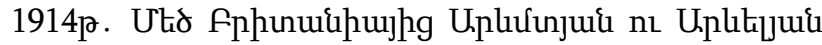

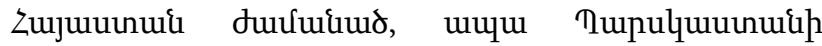
hujupiuul unupuopitip ujgtiuro úpuhnitip

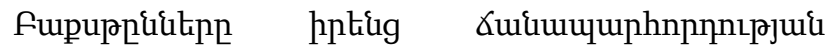

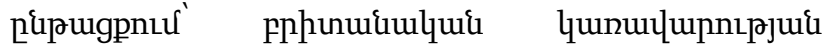

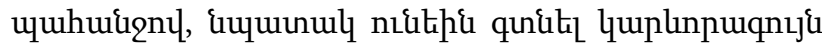

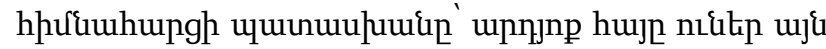

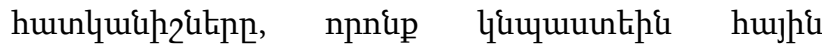

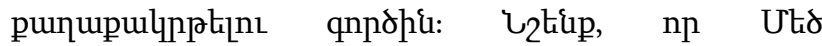

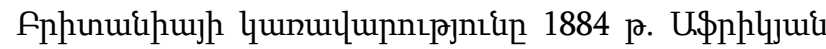

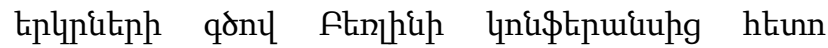

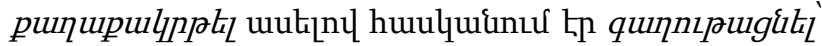

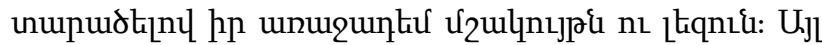

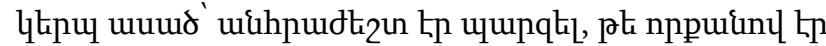

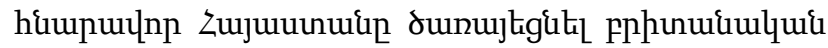
2uhhis [3, kq 26-27]:

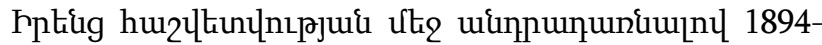

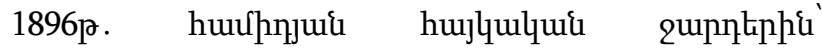

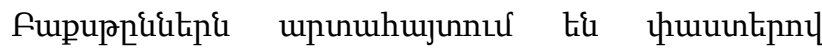

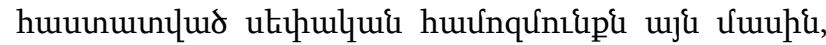

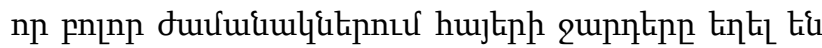

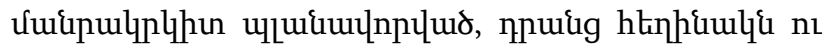

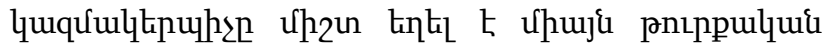

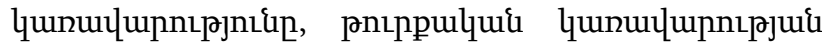

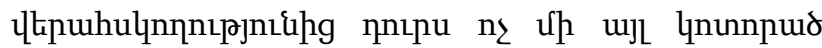

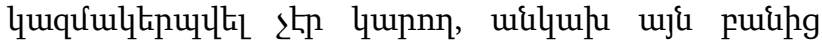

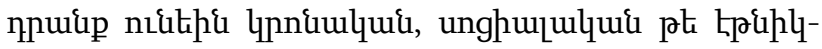

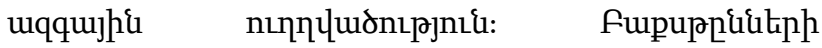

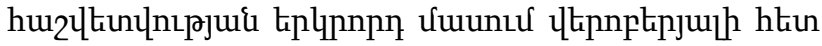

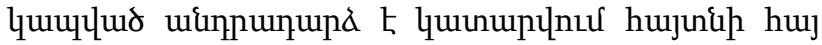

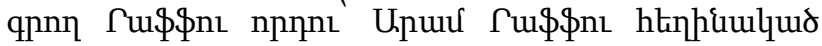
\{jnıphi:

Unuu rux $\$ \$$ ha, nl ujn 2nquintu paulylth t Lnannanud, uanpununantu t 1895 p., ntuuptipha, tepp hnppuupulqui nᄁ2 unupudurnquintu hpuqnnolnuर thi hujkph ưuuujulluw

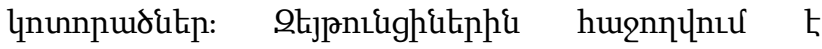

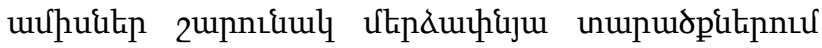
quutigiti 60000 qhiulnnitiphg punlqugur qnppn, 600

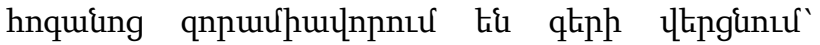

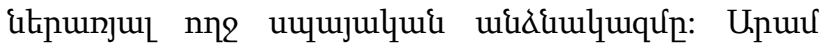

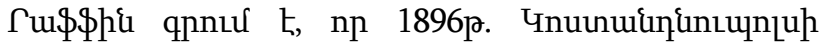

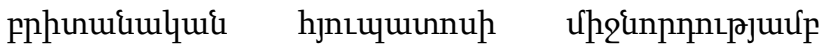

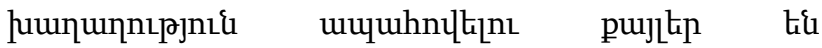

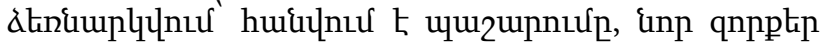

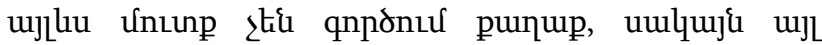

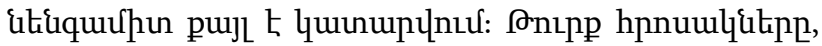

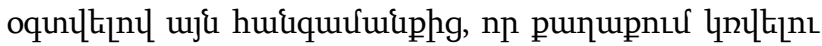

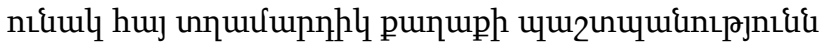
hpulquaumginud this punuph dunnuggitennud,

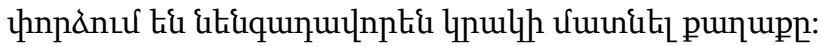

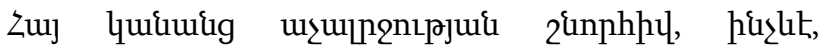

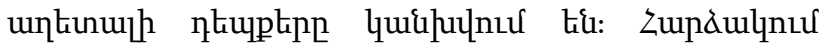

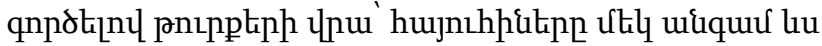

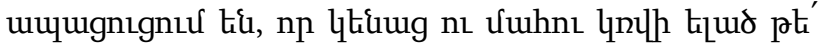

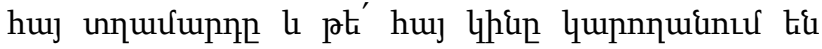

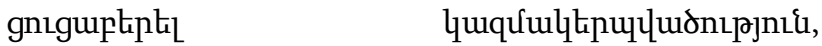

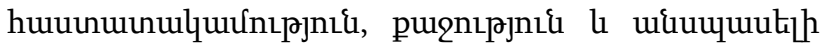

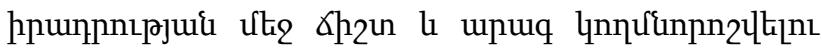

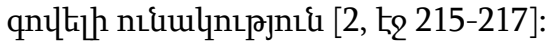

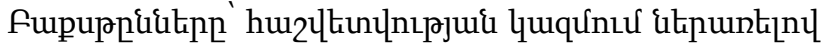

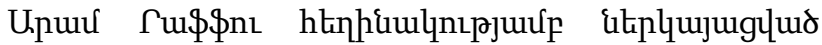

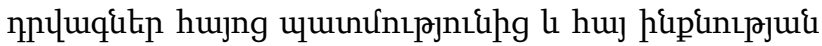

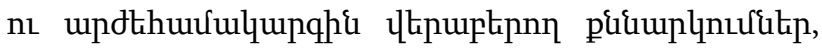

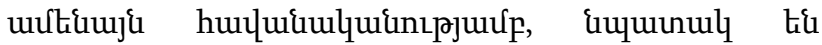

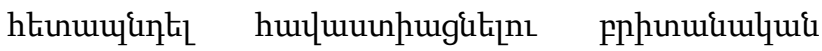
qunuulunnıpjuin $\quad \mathrm{l} \quad$ tilnnumulqui

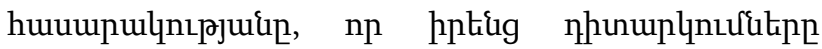

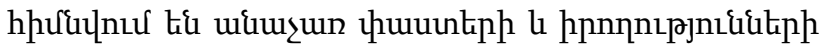

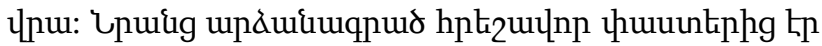

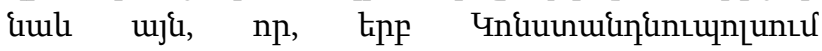

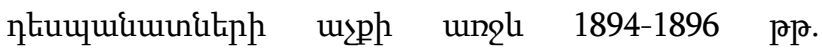

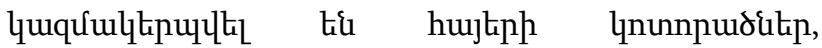
uiqqhughitiph pnpàtil tí «qpuupunupup»

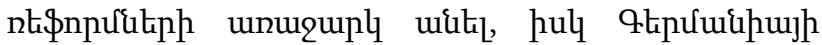

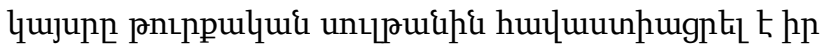

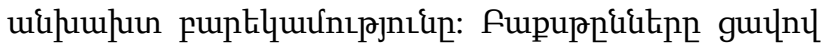
tí \&lquunnư, np tilpnuquutin tiphunpnipptipn 1908p.

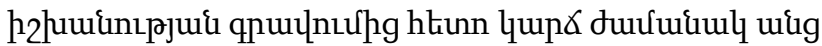

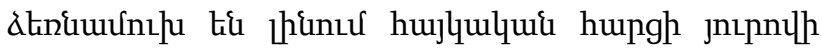




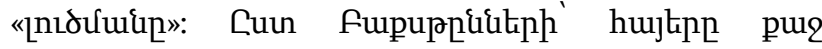

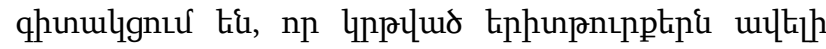

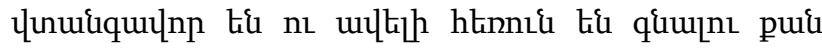

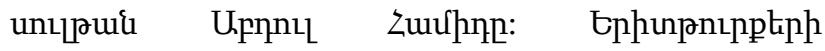

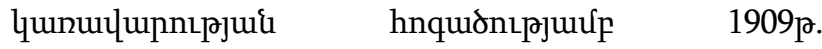

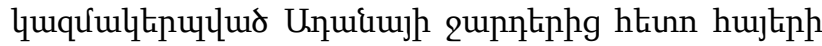

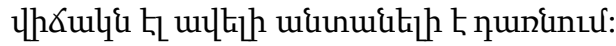

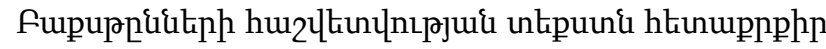
t ujf unnứnl, np npn2ulh uqnulgitiph kitppn

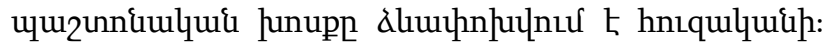

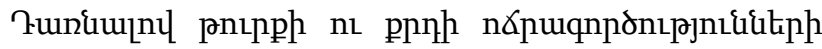

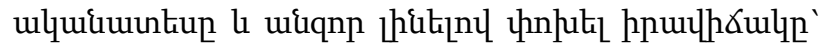
Fupupgiatipi uipupnug qujnntjpnl nt

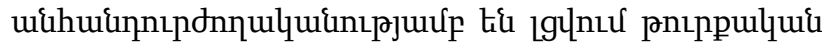

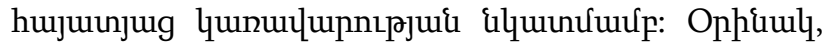

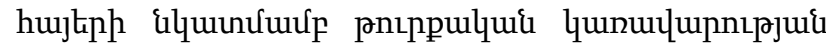

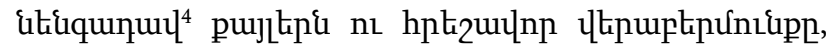

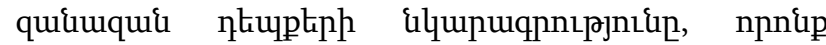

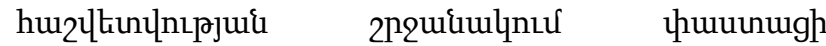

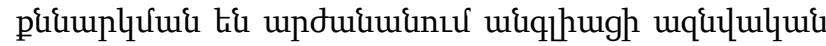

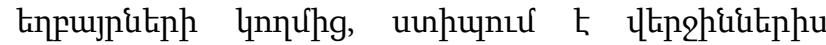

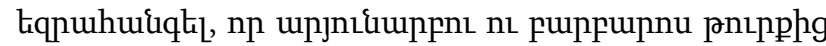

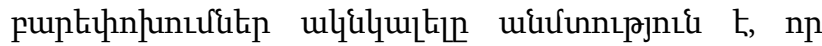

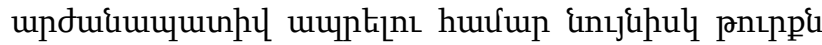

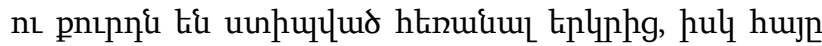

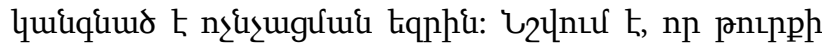

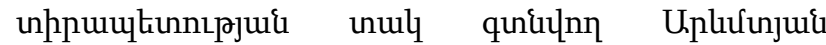

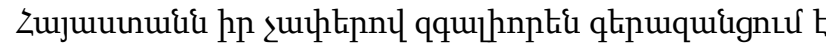

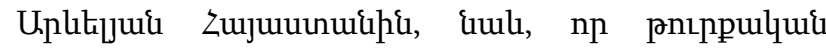

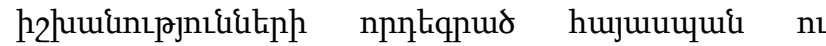

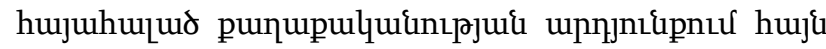

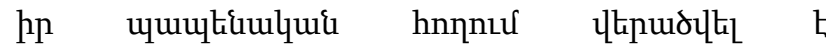
hnppuufuuunıpjuid [2, to 131]:

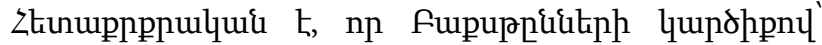

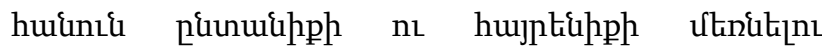

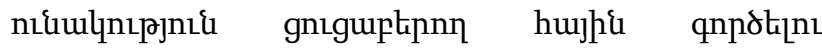

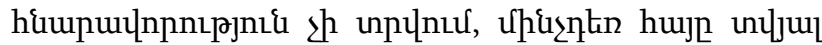

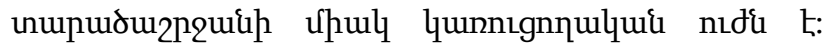

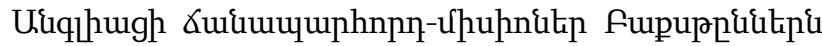

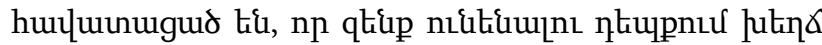

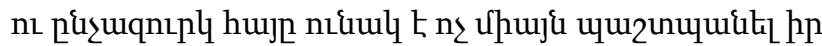

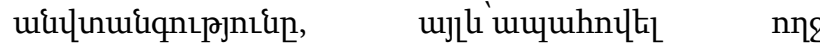

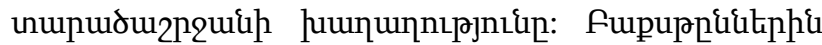

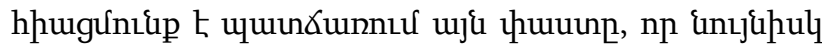

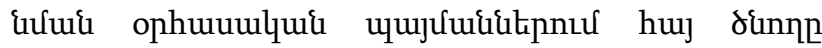

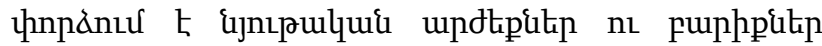

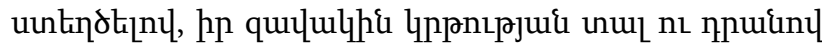
qnite uf phr ptipluugitil quululh ndilup ljuifp,

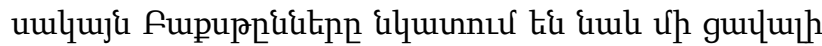

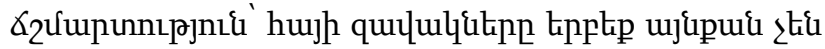

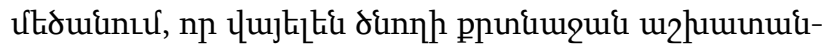

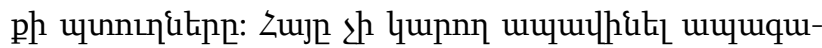

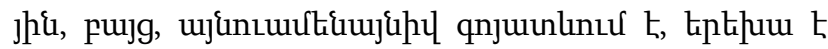

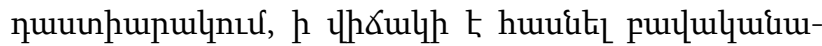

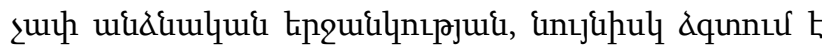

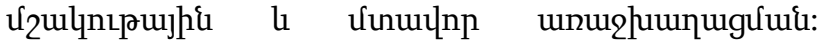

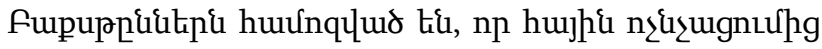

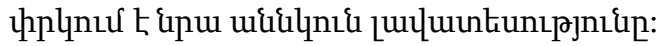

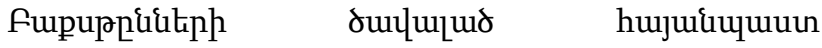

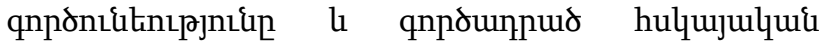

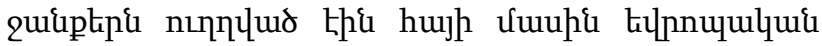

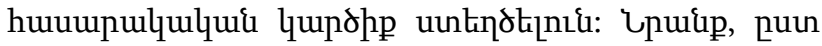

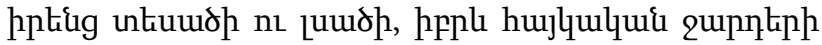

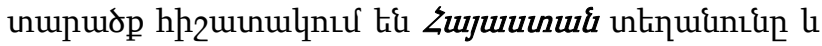

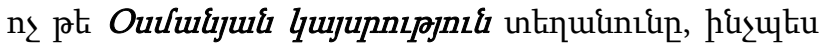

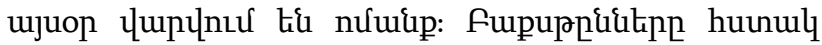

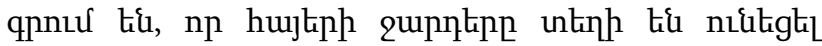
hujteph hujptipnnu, nph upnjntupnud hujp hujptiauqnlyltik:

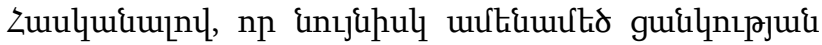

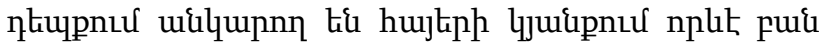

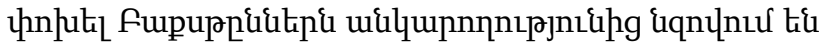

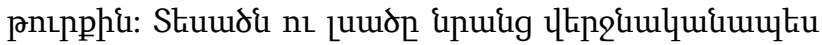

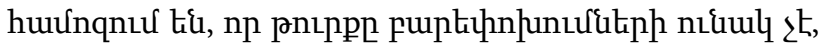

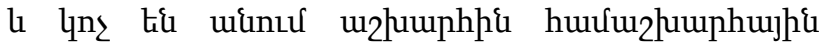

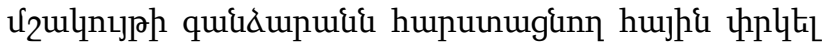

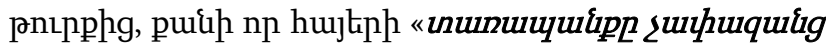

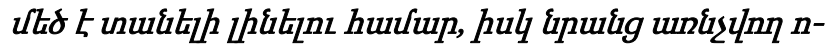

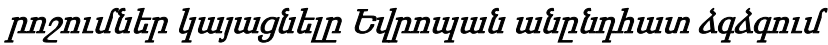

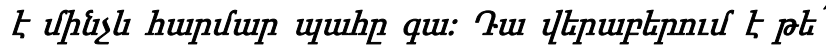

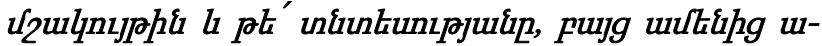

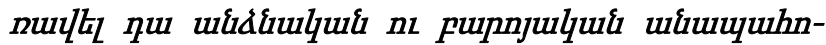

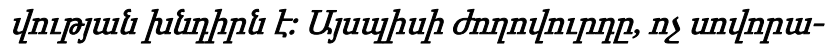

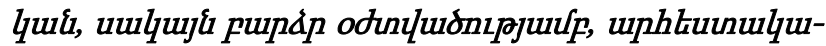

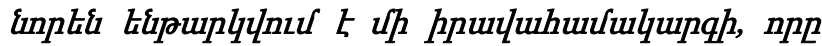

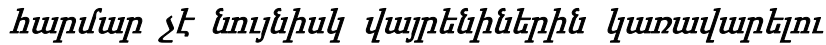

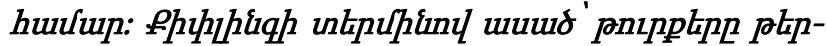

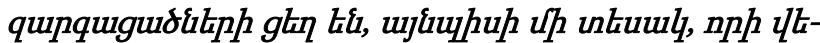

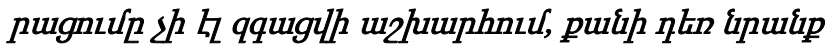

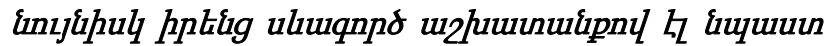

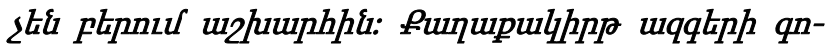

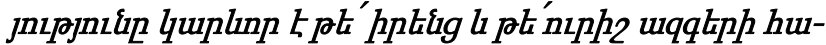

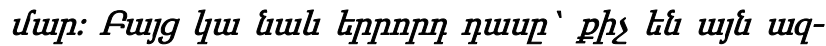

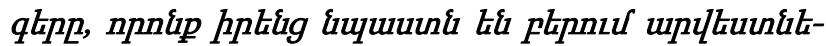

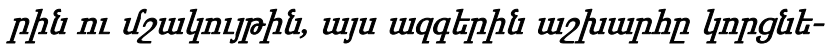

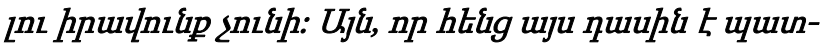

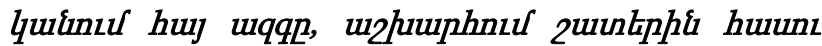
2h»[3, kq 160-161]:

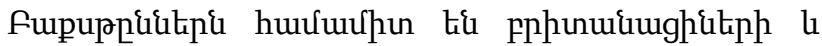

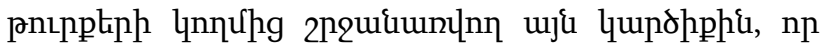

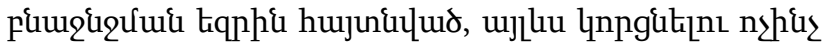

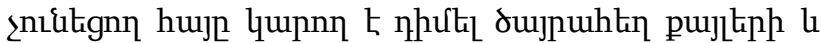

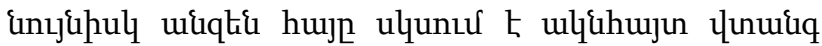

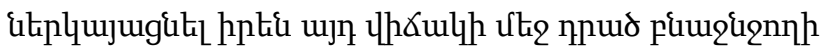
huuvun:

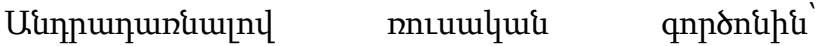

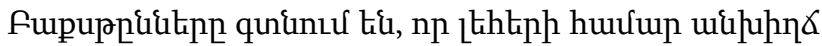

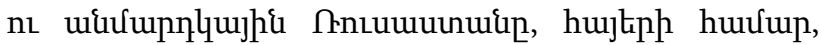

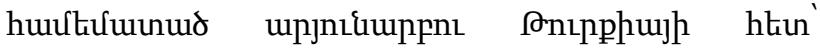
UuunLuiduinnıp हैं untuwltin, npi huj 


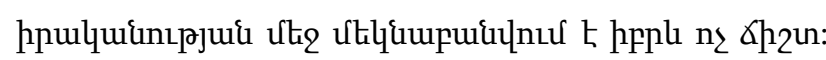

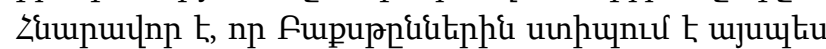

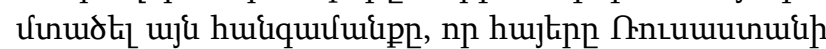

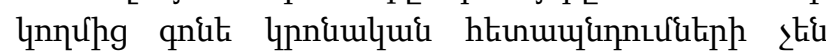
tipunlylh, ljuiph htin uihuưuuntinkth

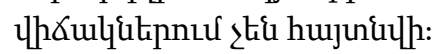

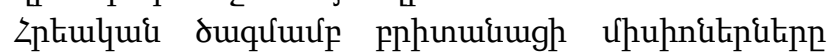

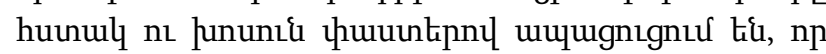

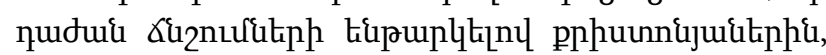

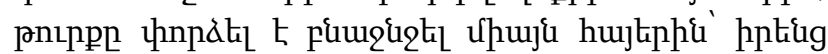

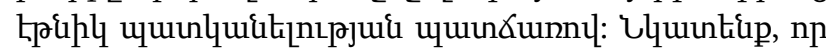

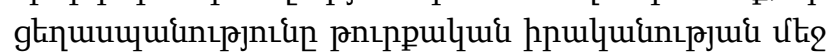

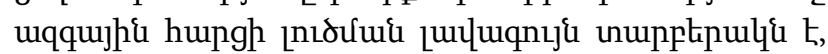

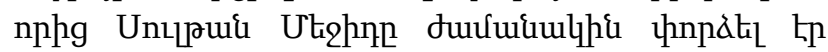
oqunlti pnnulyui hungn lnı $\delta \mathrm{t}_{1}$ hu:

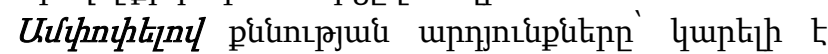

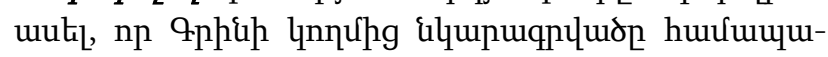

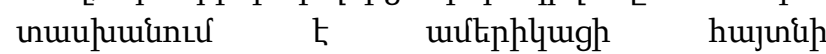

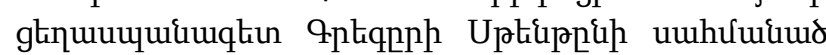

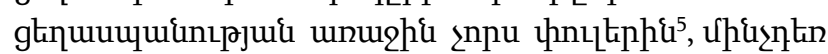

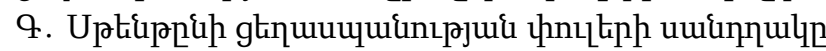

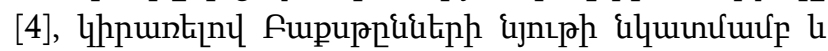

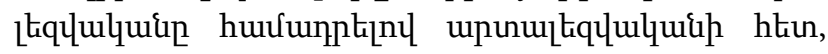
ưunqnuर tip, np ptiuptenp ulquiuuntu

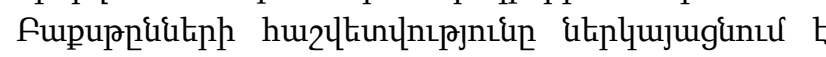
ginuuuwumıpjntukitphis

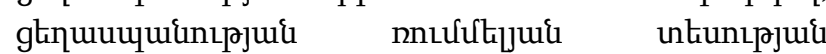

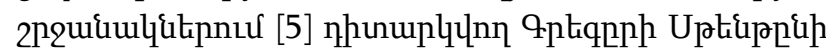

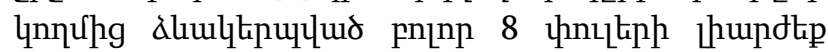

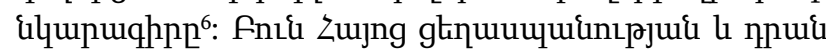

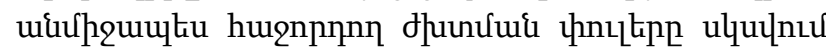
tiu 1915 p-hg:

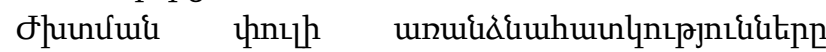

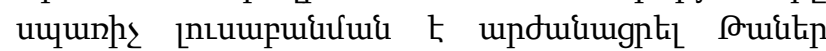
U.lॄuunp: 2016 plhi «Armenian Weekly»

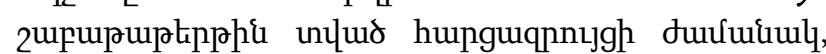

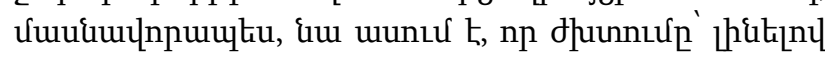

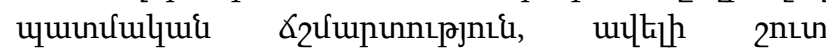

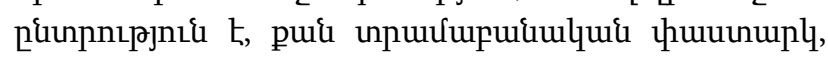
ujupipa, nppuis h np huufnqhs

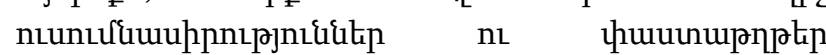

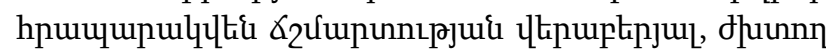

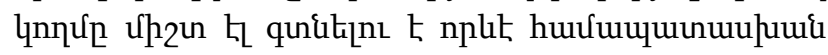
hpuuwupulnnu, nnp qukplqujugih hppl

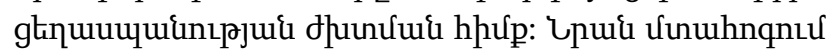

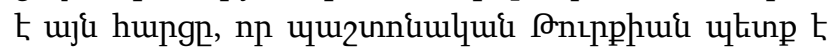

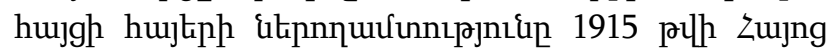

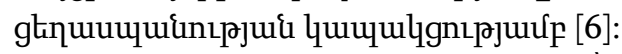

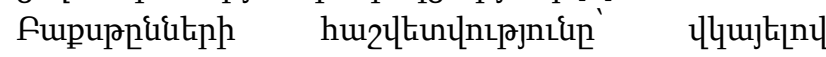

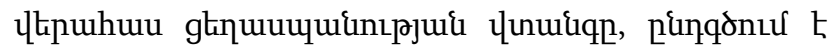

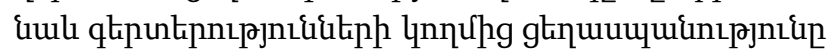

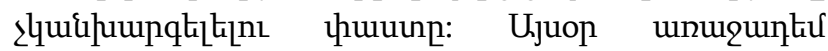

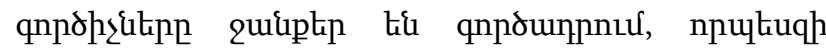

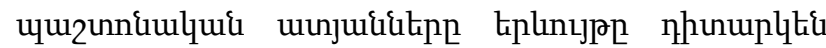

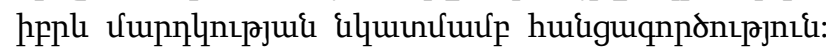

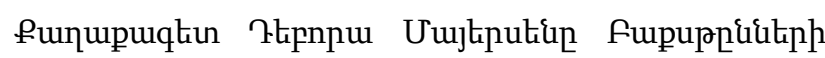

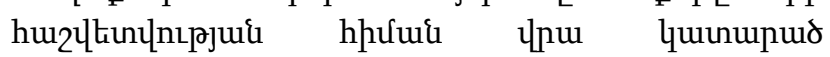

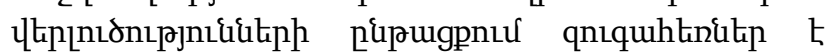

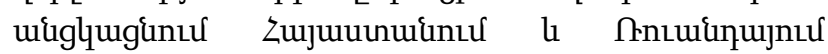

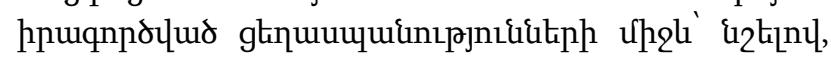

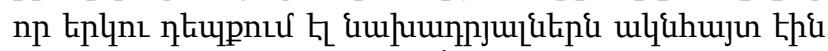
la gtnuuuwum Uujtenutias huưnqumo t, np Fupuppisitph qhppe

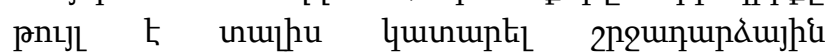

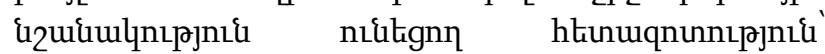

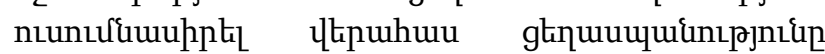

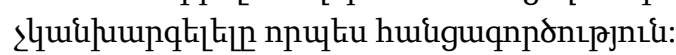

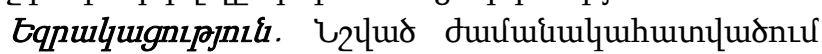

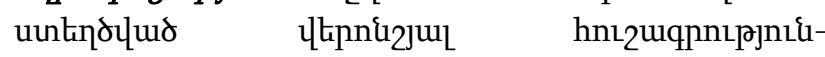

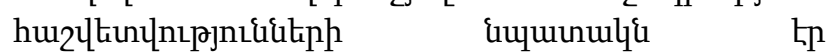

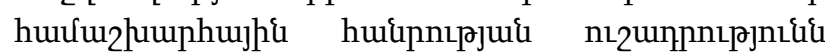

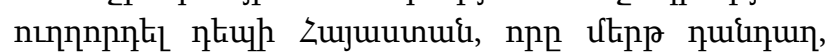

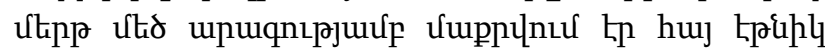

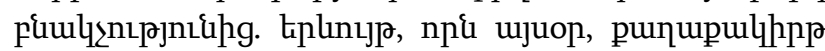

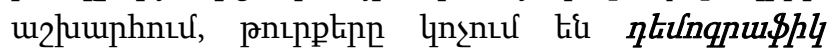

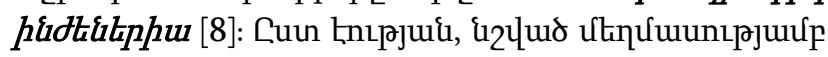

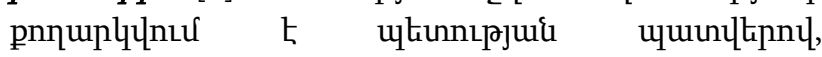

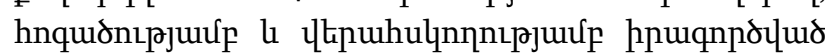

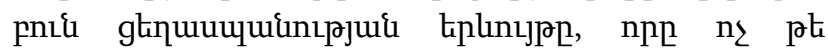

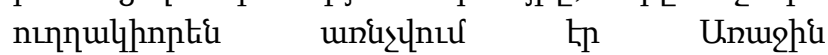

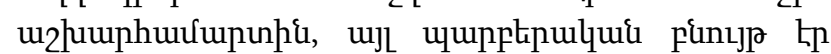

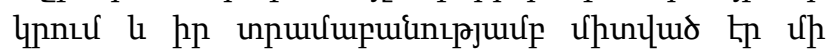

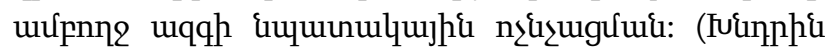

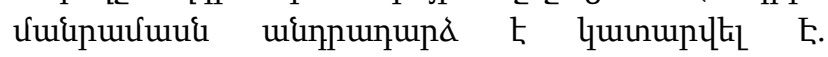

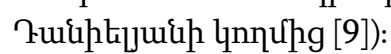

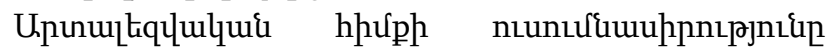

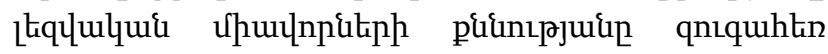
ptipnư $k$ uje hưưnqưuia, np

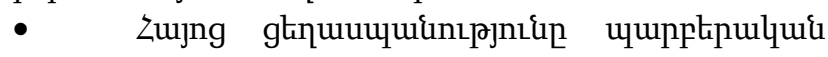

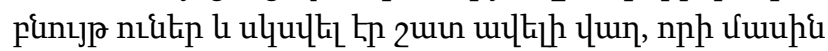

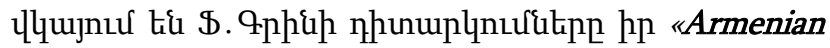
Massacres and Turkish Tyranny» hnı̨uqpnıрjuil utiq.

- Fnhunuiulquí qunuulunnıpjuian 1914p. ¿itpluujugnuo "Travel and politics in Armenia»

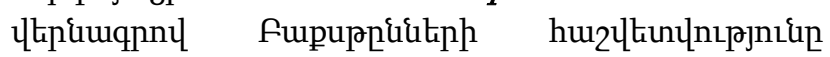
uighpudtizu $t$ nhunupllil hppl zujng

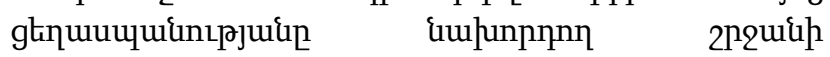

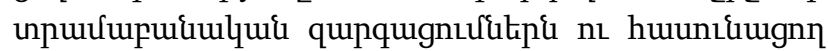

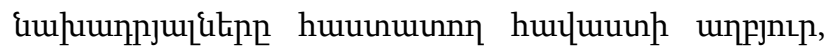
nuin nph 1915p. Uplưunjuí Zujuuunuintu untnh

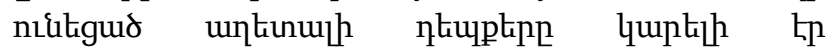
quiduuuntiuti].

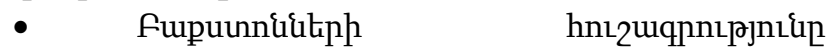

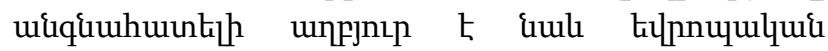

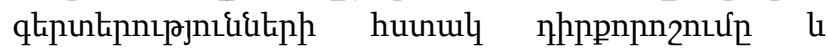

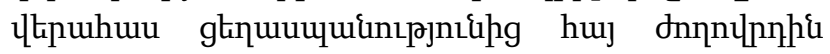

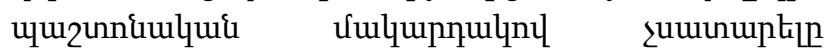

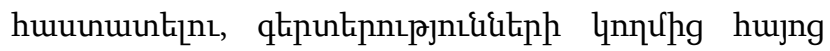

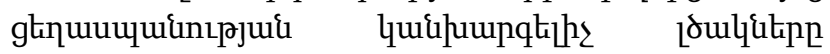

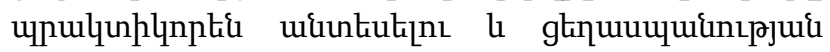




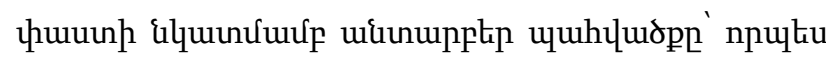

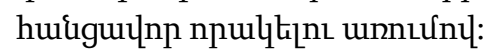

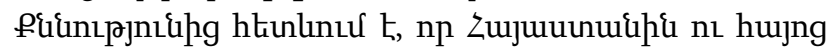

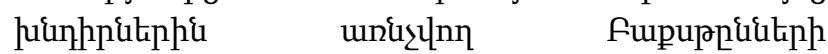

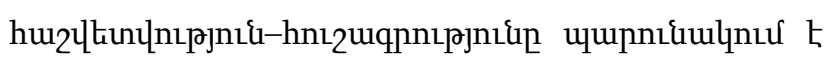

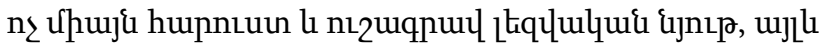
oquntu $t$ puguhujutis quplenp uquendulquil

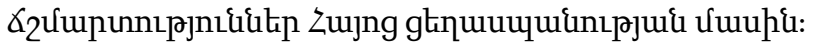

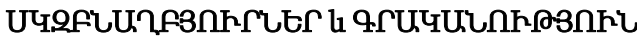 w) U५.2FนUาค3กคГนเก}

1. Greene Frederick (1896) Armenian Massacres and Turkish Tyranny. Philadelphia \& Chicago, Inter.Pub. Co. //Available at:

https://archive.org/details/armenianmassacre00greeiala/page/n 6>. [Accessed October 2018].
2. Buxton N. and H. Buxton (1914)Travel And Politics in Armenia, London: Smith Elder \&CO. Waterloo Place, 14. //Available

$<$ https://archive.org/details/travelpoliticsin00noel/page/n8>. [Accessed October 2018].

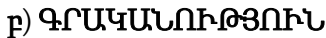

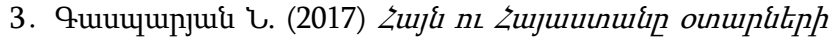
urplipnц, Epluui, ๒T之 hpuun. Available at: $<$ http://publishing.ysu.am/files/HaynuHayastany_otarneri_ach qerov.pdf $>$. [Accessed June 2019].

4. Stanton G. President, Genocide Watch. The Ten Stages of Genocide. Available at: <www.genocide watch.org/images/8StagesBriefingpaper.pdf >. [Accessed May 2018].

5. Rummel R. (1997) Statistics of Democide. Charlottesville. // Available at: <https:// www. hawaii.edu/power kills/GENOCIDE.HTM>. [Accessed March 2016].

6. Akçam T. (2016) The Authenticity of the Naim Efendi Memoirs and Talat Pasha Telegrams. Available at: $<$ http://asbarez.com/155718/akcam-the-authenticity-of-thenaim-efendi-memoirs-and-talat-pasha-telegrams/>. [Accessed October 2017].

7. Mayersen D. (2014). On the Path to Genocide: Armenia and Rwanda Reexamined, N.Y., Berghahn books.

8. Şeker N. (2013) Forced Population Movements in the
Ottoman Empire and the Early Turkish Republic: An Attempt at Reassessment through Demographic Engineering // EJTS, 16. Available at: <https://ejts.revues.org/439>. [Accessed March 2018].

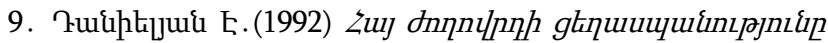

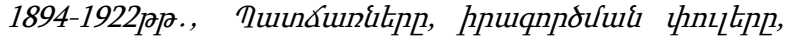

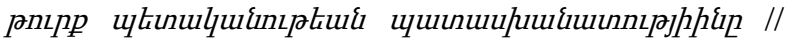

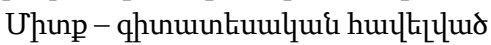

10. Gasparyan N., Zohrabyan Ed. (2017) Linguocognitive Approach To Teaching And Interpretation of Armenian Genocide Issues. // Foreign Languages in Higher Education, 2 (23), pp.213-225, Yerevan: YSU Press. // Available at: <http://ysu.am/files/9-1544603494-.pdf >[Accessed May 2019].

11. Poghosyan V. (2014) The Echoes of the Armenian Massacres of Cilicia in "The Times". // Armenological Issues, Bulletin 1, pp.159-164, Yerevan: YSU Press.

12. Astvatsaturian Turcotte Anna, (2012) Nowhere: A Story of Exile Lexington, MA, USA, Publ. Tatoul Sonentz-Papazian.

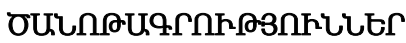

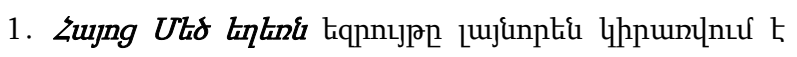

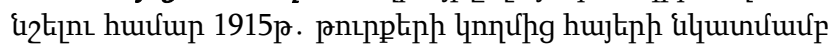

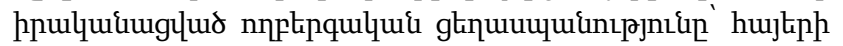

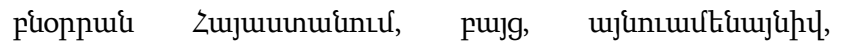

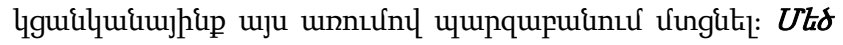

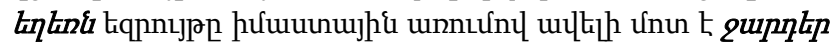

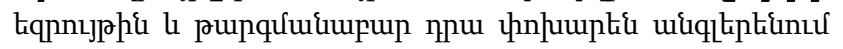

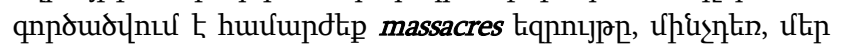

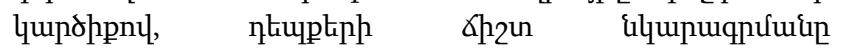

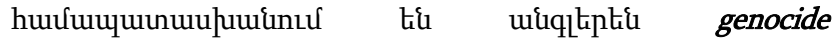

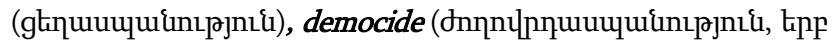

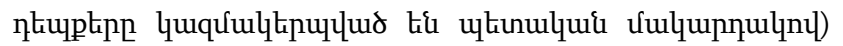
tinnujpilinn [10, ts 213-225]:

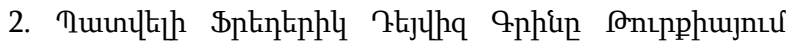

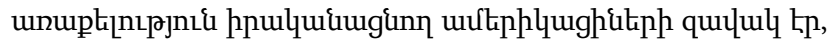

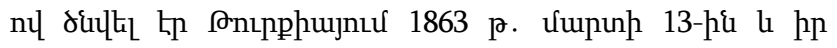

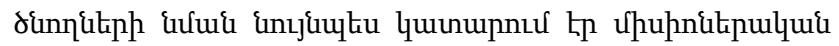

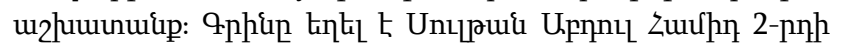

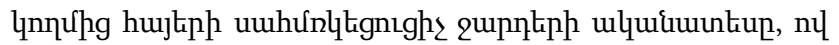

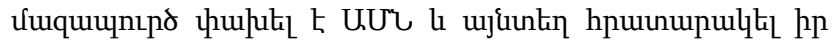

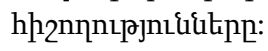

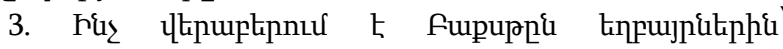

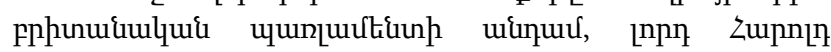

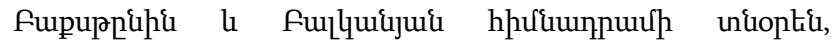

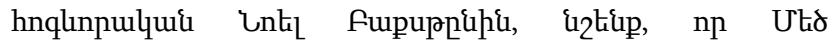

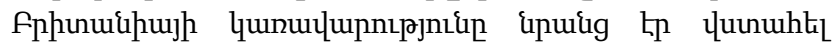

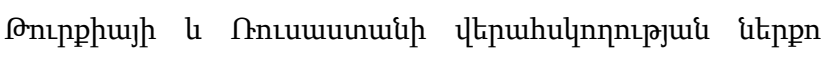

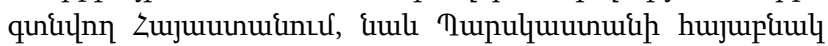

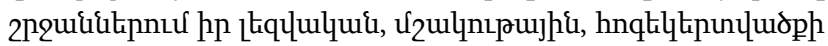

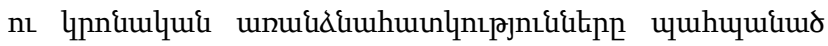

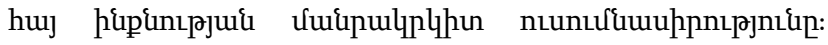

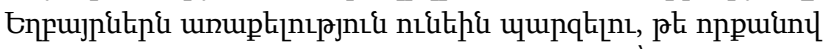

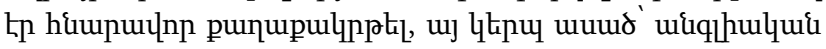
zuhpi dunujtigiti hujha: Fupupnkatiph

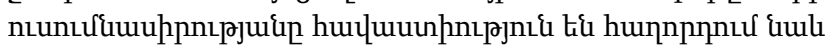

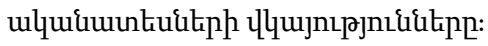

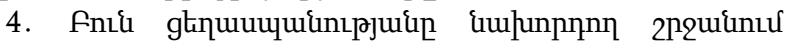

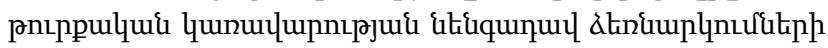

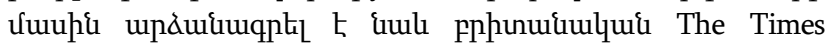

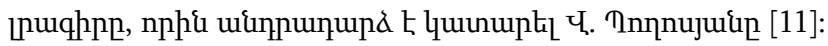

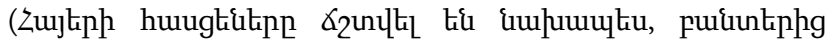

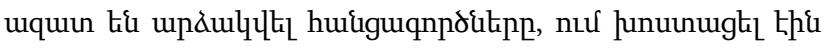

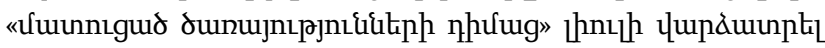

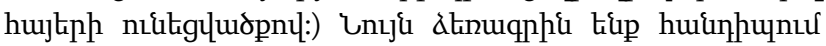

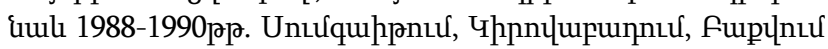

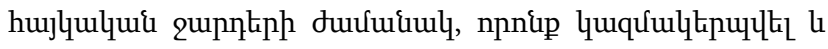

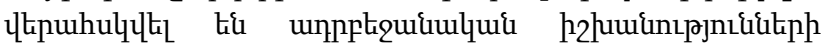
lnnuhg [12]:

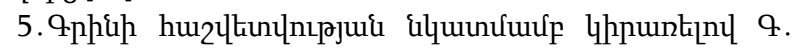

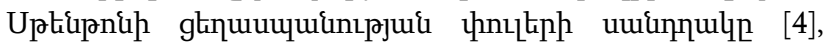

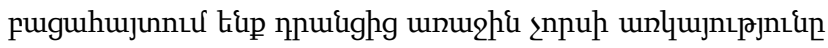

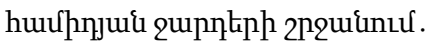




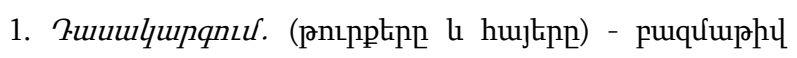

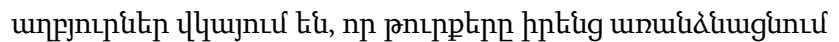

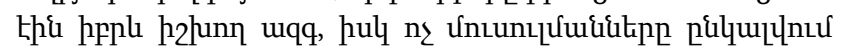

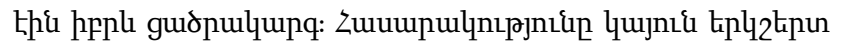
Łn.

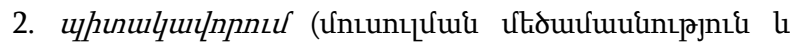

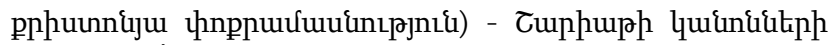

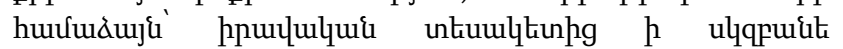

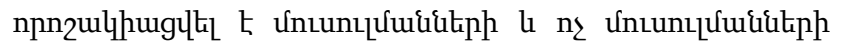

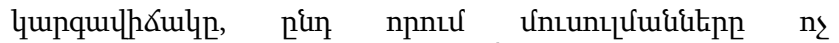

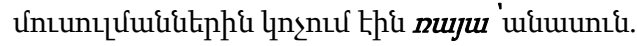

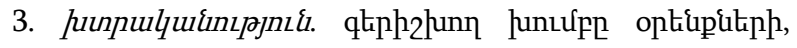
unlnpnujpiliph li punupulqui nıdp lhpunưurf

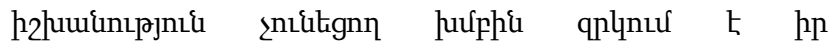
punupughulqui hpulntapdatphg, nunquhuplnud tid

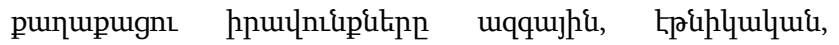

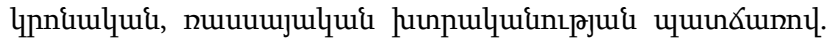

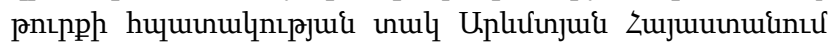

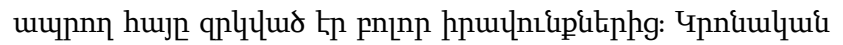

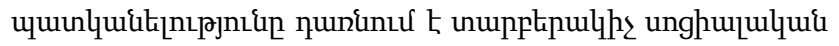

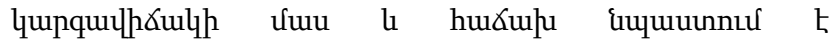

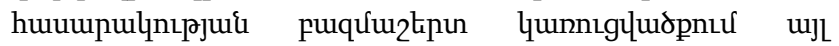

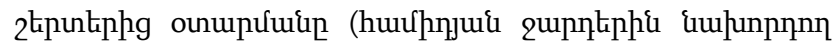
212uil).

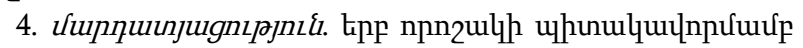

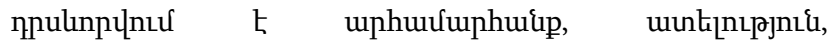

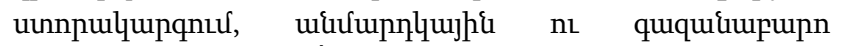

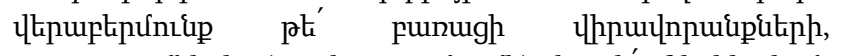

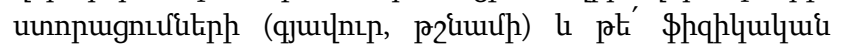

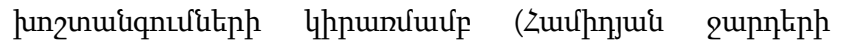
212uila).

\section{Memoir-Accounts Of Foreigners As Valuable Sources In The study Of Armenian Genocide History \\ N. Gasparyan, A. Darbinyan}

Abstract. The article discusses urgent problems dealing with Armenian linguo-culture and national issues. Memoir-accounts written for the British Government are investigated as valuable sources in the study of Armenian Genocide history. The authors were expected to present valid and impartial details on Armenia and Armenians without distorting the facts. The textological analysis of foreign missionaries' speeches is carried out from the linguo-cognitive perspective with the account of the ex tra-linguistic reality. The method of careful sampling of examples is applied.

Keywords: Memoir-accounts, sources, Armenian, Turkish massacres, genocide.

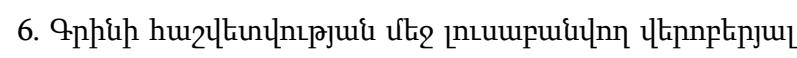

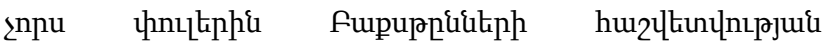

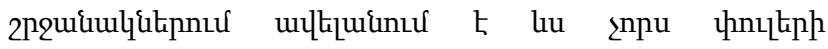
\{lyupuqpnıрjnı\&.

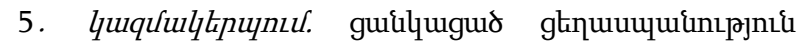

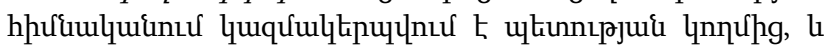

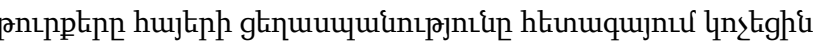

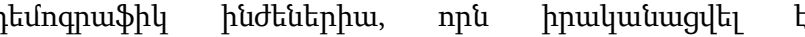

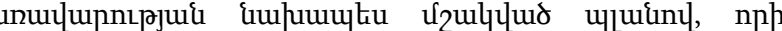

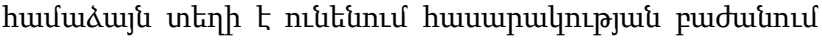

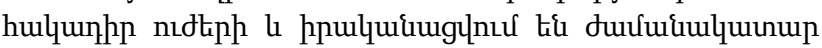
quipuuuuunpuuunnıpjniditip.

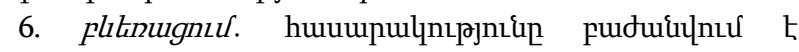

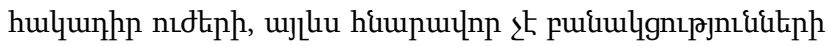

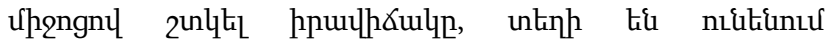

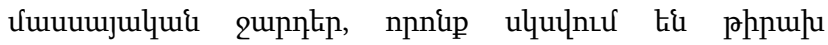

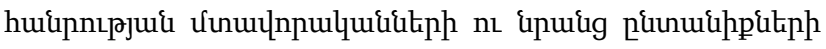
uuquinıpjnıfiatipnц.

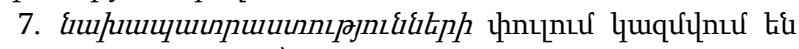
qnhtiph gnigulqutip nuu uqqujhi hipanıpjuis.

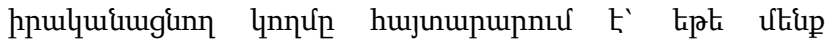

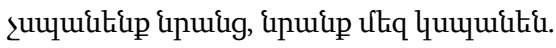

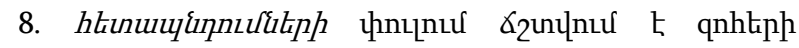

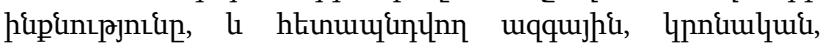

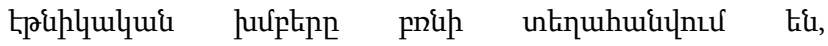

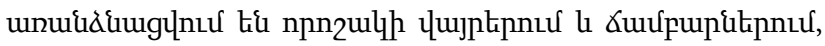

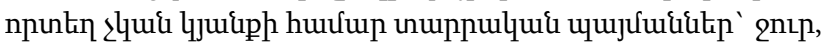

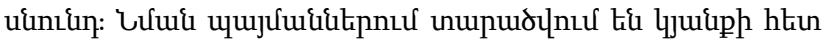

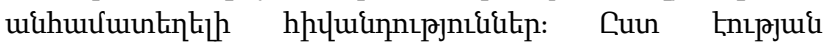

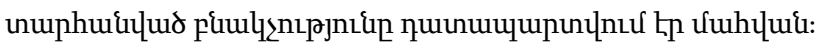

